\title{
CHEBYSHEV SUBSPACES AND CONVERGENCE OF POSITIVE LINEAR OPERATORS
}

\author{
C. A. MICCHELLI ${ }^{1}$
}

\begin{abstract}
A theorem of Korovkin states that a sequence of positive linear operators on $C[a, b]$ converges strongly to the identity if and only if convergence holds on a three-dimensional Chebyshev subspace of $C[a, b]$. We extend this theorem to include Chebyshev subspaces of arbitrary dimension and convergence to other positive linear operators.
\end{abstract}

1. Introduction. Convergence of a sequence of positive linear operators to the identity can sometimes be proven by verifying convergence on a finite set of functions. For instance, let $C(X)$ denote the Banach space of real-valued functions which are defined and continuous on the interval $X=[a, b]$. Then a sequence of positive linear operators on $C(X)$ converges strongly to the identity provided convergence holds on a three-dimensional Chebyshev subspace of $C(X)$. This well known and striking result is due to Korovkin (cf. [4]).

There is another formulation of this theorem in terms of linear functionals on $C(X)$. Given an $x \in X$, we define point evaluation at $x$ as the linear functional $\hat{x}(f)=f(x)$. A sequence of positive linear functionals $\left\{L_{k}\right\}$ converges weakly to $\hat{x}$, that is,

$$
\lim _{k \rightarrow \infty} L_{k}(f)=\hat{x}(f), \quad f \in C(X),
$$

if and only if (1) holds for a three-dimensional Chebyshev subspace of $C(X)$.

The purpose of this paper is to extend this theorem to sequences of positive linear functionals converging to linear functionals other than point evaluations. Specifically, we answer the following question: What linear functionals $L$ have the property that a sequence of positive linear functionals converges weakly to $L$ on $C(X)$ if and only if convergence holds on an $n+1$-dimensional Chebyshev subspace of $C(X)$. We prove the corresponding result for positive linear operators, show that certain improvements in our result are not possible, and give some examples.

Received by the editors August 21, 1972 and, in revised form, January 8, 1973.

AMS (MOS) subject classifications (1970). Primary 41 A35.

Key words and phrases. Chebyshev subspace, positive operators, Korovkin's theorem.

${ }^{1}$ This paper is based largely on the author's doctoral dissertation written under the supervision of Professor S. Karlin.

(c) American Mathematical Society 1973 
For further generalizations of Korovkin's theorem see [5] or [6].

2. Convergence of positive linear operators. Consider the following positive linear functional

$$
L(f)=\sum_{i=1}^{k} \lambda_{i} f\left(x_{i}\right)=\sum_{i=1}^{k} \lambda_{i} \hat{x}_{i}(f),
$$

$\lambda_{i}>0, i=1, \cdots, k$ and $a \leqq x_{1}<\cdots<x_{k} \leqq b$. Let us assign a weight of one to an $x_{i}$ which lies in the interior of the interval $[a, b]$, while an endpoint, if it appears in (2), will be given a weight of $\frac{1}{2}$. The sum of these weights is called the index of $L$ and will be denoted by $I(L) . \mathscr{L}_{n}$ is defined to be the space of all positive linear functionals of the form (2) which have index $\leqq n / 2$. An $n$-dimensional linear subspace $\mathscr{U}$ of $C(X)$ is called a Chebyshev subspace if every nonzero element of $\mathscr{U}$ has at most $n-1$ zeros in $X$.

The basic ingredient in the proof of the Bohman-Korovkin theorem is the existence of nonnegative functions which have a prescribed set of zeros. The following result, whose proof can be found in [2], provides us with this information for an arbitrary Chebyshev subspace.

THEOREM A. Let $\mathscr{U}$ be a $n+1$-dimensional Chebyshe: subspace of $C(X)$ and suppose $S=\left\{s_{1}, s_{2}, \cdots, s_{k}\right\}$ is a set of $k$ dist inct points in $[a, b]$ such that $\sum_{i=1}^{k} w\left(s_{i}\right) \leqq n$ where $w(s)$ is defined to be two if $s \in(a, b)$ and one otherwise. Then there exists a nontrivial nonnegative function $u \in \mathscr{U}$ which vanishes precisely at the points of $S$. The only exception is that if $n=2 m$ and exactly one of the endpoints $a$ or $b$ is in $S$, then $u$ may vanish at the other endpoint as well.

THEOREM 1. Let $\mathscr{U}$ be an $n+1$-dimensional Chebyshev subspace of $C(X)$. A sequence of positive linear functionals $\left\{L_{k}\right\}$ on $C(X)$ converges weakly to an $L \in \mathscr{L}_{n}$ if and only if

$$
\lim _{k \rightarrow \infty} L_{k}(u)=L(u), \quad u \in \mathscr{U} .
$$

Proof. Assume that (3) holds. Since $\mathscr{U}$ is a Chebyshev subspace, there exists a $v \in \mathscr{U}$ which is positive on the interval $[a, b]$ (Theorem A). Set $m=\min _{x \in X} v(x)$, then in view of the positivity of $L_{k}$

$$
\left\|L_{k}\right\|=L_{k}(1) \leqq L_{k}(v) / m .
$$

Here, $\left\|L_{k}\right\|$ denotes the norm of the linear functional $L_{k}$. Since $\lim _{k \rightarrow \infty} L_{k}(v)$ exists, we conclude from (3) that $\operatorname{Sup}_{k}\left\|L_{k}\right\|$ is finite. The Helly selection theorem (cf. [1, Theorem 4.12.3]) states that any sequence of norm bounded linear functionals on $C(X)$ has a weakly convergent subsequence. Thus we conclude that $\left\{L_{k}\right\}$ has a weakly convergent subsequence. 
Let $L_{0}$ denote any weak cluster point of the sequence $\left\{L_{k}\right\}$. The proof will be complete if we can show $L_{0}=L$. To this aim, we first note that $L_{0}$ is necessarily a positive linear functional which, on account of (3), satisfies

$$
L_{0}(u)=L(u), \quad u \in \mathscr{U} .
$$

Furthermore, since $L \in \mathscr{L}_{n}, L$ can be represented in the form

$$
L=\sum_{i=1}^{k} \lambda_{i} \hat{x}_{i}, \quad \lambda_{i}>0
$$

with $I(L) \leqq n / 2$. There exists, according to Theorem A, a nonnegative $\bar{u} \in \mathscr{U}$ which vanishes only at $x \in\left\{x_{1}, \cdots, x_{k}\right\}$ or possibly at an endpoint of $[a, b]$ not in $\left\{x_{1}, \cdots, x_{k}\right\}$. Therefore, from (5), (6) and the positivity of $L_{0}$, we conclude that

$$
L_{0}=\sum_{i=0}^{k} \mu_{i} \hat{x}_{i}
$$

where $\mu_{i} \geqq 0, i=0,1, \cdots, k$ and $x_{0} \in\{a, b\}$ (if $n \neq 2 m$ or both endpoints are already in the set $\left\{x_{1}, \cdots, x_{k}\right\}$, then $\mu_{0}$ can be taken to be zero).

Substituting this expression for $L_{0}$ into (5), we obtain the relation

$$
\sum_{i=0}^{k}\left(\lambda_{i}-\mu_{i}\right) \hat{x}_{i}(u)=0, \quad u \in \mathscr{U}
$$

where we have defined $\lambda_{0}=0$. Since $I(L) \leqq n / 2$, there are no more than $n+1$ summands in (8) and so it follows that $\lambda_{i}=\mu_{i}, i=0,1, \cdots, k$.

Remark. Theorem 1 implies that the restriction of any $L \in \mathscr{L}_{n}$ to an $n+1$-dimensional Chebyshev subspace has a unique extension as a positive linear functional on $C(X)$.

We now turn our attention to the version of Theorem 1 which is valid for positive linear operators. Let $T$ be a linear operator on $C(X)$. For each $x \in X$, we define the linear functional $\hat{x} \circ T$ by setting $(\hat{x} \circ T) f=(T f)(x)$.

THEOREM 2. Let $T$ be a positive linear operator on $C(X)$ such that $\hat{x} \circ T \in \mathscr{L}_{n}$ for each $x \in X=[a, b]$. Then a sequence of positive linear operators $T_{k}$ converges strongly to $T$ if and only if

$$
\lim _{k \rightarrow \infty} T_{k} u=T u, \quad u \in \mathscr{U},
$$

where $\mathscr{U}$ is some $n+1$-dimensional Chebyshev subpace of $C(X)$.

Proof. Suppose to the contrary that there exists a $g \in C(X)$ such that $T_{k} g$ does not converge to $T g$ while (9) holds. Then there exists an $\varepsilon_{0}>0$, $n_{k} \rightarrow \infty$ and a sequence $\left\{x_{k}\right\} \subseteq X$ such that

$$
\left|\left(\hat{x}_{k} \circ T_{n_{k}}\right) g-\left(\hat{x}_{k} \circ T\right) g\right| \geqq \varepsilon_{0} .
$$


Since $X$ is compact, the sequence $\left\{x_{k}\right\}$ has a convergent subsequence. We also denote this subsequence by $\left\{x_{k}\right\}$ and its limit by $x_{0}$. Then from (9) we have $\lim _{k \rightarrow \infty}\left(\hat{x}_{k} \circ T_{n_{k}}\right) u=\left(\hat{x}_{0} \circ T\right) u$, for all $u \in \mathscr{U}$. Hence, it follows from Theorem 1 that $\lim _{k \rightarrow \infty}\left(\hat{x}_{k} \circ T_{n_{k}}\right) g=\left(\hat{x}_{0} \circ T\right) g$. This contradicts (10) and so the theorem is proven. by

Example. Let $f \in C[0,1]$. The $n$th Bernstein polynomial of $f$ is defined

$$
\left(B_{n} f\right)(x)=\sum_{k=0}^{n}\left(\begin{array}{l}
n \\
k
\end{array}\right) x^{k}(1-x)^{n-k} f\left(\frac{k}{n}\right) .
$$

Note that $B_{n}$ is a positive linear operator on $C[0,1]$. Moreover, if we define $u_{0}(x)=1, u_{1}(x)=x$, and $u_{2}(x)=x(1-x)$ then the following equations can be verified

$$
B_{n} u_{0}=u_{0}, \quad B_{n} u_{1}=u_{1}, \quad \text { and } \quad B_{n} u_{2}=(1-1 / n) u_{2}
$$

(cf. [5]).

Let $B_{n}^{k}$ denote the $k$ th power of the linear operation $B_{n}$ and define

$$
\begin{array}{ll}
T f=f, & \text { if } \lim _{n \rightarrow \infty} \frac{k_{n}}{n}=0, \\
T f=B_{1} f, & \text { if } \lim _{n \rightarrow \infty} \frac{k_{n}}{n}=\infty .
\end{array}
$$

Then from (11) it immediately follows that $\lim _{n \rightarrow \infty} B_{n}^{k_{n}} Q \rightarrow T Q$ for any quadratic polynomial $Q$. Since $\hat{x} \circ T \in \mathscr{L}_{2}$ for every $x \in[0,1]$ we can conclude from Theorem 2 that $\lim _{n \rightarrow \infty} B_{n}^{k_{n}} f \rightarrow T f$ for all $f \in C[0,1]$. This fact appears in [3].

3. Improving Theorem 1. We will show that Theorem 1 is not valid if either the hypothesis on $L$ or the subspace $\mathscr{U}$ is removed.

THEOREM 3. Suppose $\mathscr{U}$ is an $n+1$-dimensional Chebyshev subspace of $C(X)$. Let $L$ be a positive linear functional on $C(X)$ with the property that its restriction to $\mathscr{U}$ has a unique extension as a positive linear functional on $C(X)$. Then $L$ must be in $\mathscr{L}_{n}$.

Proof. Assume to the contrary that $L \notin \mathscr{L}_{n}$ and let $\left\{u_{0}, \cdots, u_{n}\right\}$ be a basis for the Chebyshev subspace $\mathscr{U}$. Consider the moment space $\mathscr{M}_{n+1}$ generated by the set of functions $\left\{u_{0}, u_{1}, \cdots, u_{n}\right\}$,

$$
\mathscr{M}_{n+1}=\left\{c=\left(c_{0}, c_{1}, \cdots, c_{n}\right) \in E^{n+1} \mid c_{i}=F\left(u_{i}\right), i=0,1, \cdots, n\right\},
$$

where $F$ ranges over all positive linear functionals on $C(X)$. In [2] it is proven that $\mathscr{M}_{n+1}$ is a closed convex cone in $E^{n+1}$ whose boundary is 
precisely the set

$$
\left\{c=\left(c_{0}, \cdots, c_{n}\right) \in E^{n+1} \mid c_{i}=F\left(u_{i}\right), i=0,1, \cdots, n, F \in \mathscr{L}_{n}\right\} .
$$

Thus, the vector $c=\left(L\left(u_{0}\right), \cdots, L\left(u_{n}\right)\right)$ lies in the interior of $\mathscr{M}_{n+1}$. But for every interior point of $\mathscr{M}_{n+1}$ there exist exactly two positive linear functionals $F_{1}$ and $F_{2}$ in $\mathscr{L}_{n+1}$ which represent $L$, that is, for which we have $F_{1}(u)=F_{2}(u)=L(u), u \in \mathscr{U}$. (This result also appears in [2]. $F_{1}$ and $F_{2}$ are referred to as principal representations of $L$.) This is a contradiction and so $L$ must be in $\mathscr{L}_{n}$.

THEOREM 4. Suppose $\mathscr{U}$ is an $n+1$-dimensional subspace of $C(X)$ which is not a Chebyshev subspace, then there exist positive linear functionals $R$ and $L$ on $C(X)$ such that $L \in \mathscr{L}_{n}, R u=L u$, for all $u \in \mathscr{U}$ and $R \neq L$.

Proof. Let $\left\{u_{0}, u_{1}, \cdots, u_{n}\right\}$ be a basis for the subspace $\mathscr{U}$. Since $\mathscr{U}$ is not a Chebyshev subspace, there exist points $t_{0}<t_{1}<\cdots<t_{n}$ such that the system of equations

$$
\sum_{i=0}^{n} \lambda_{i} u_{j}\left(t_{i}\right)=0, \quad j=0,1, \cdots, n,
$$

has a nonzero solution. We assume without loss of generality that $\left|\lambda_{i}\right|<1$, $i=0,1, \cdots, n$ and the set $J=\left\{j: \lambda_{j}>0\right\}$ has cardinality $\leqq[n / 2]$. Define $L=\sum_{j \in J} \lambda_{j} \hat{t}_{j}$ and $R=-\sum_{j_{\ddagger} J} \lambda_{j} \hat{t}_{j}$. Then (12) implies $L u=R u$, for all $u \in \mathscr{U}$. Moreover, $L \in \mathscr{L}_{n}, L \neq R$ and $R$ is a positive linear functional. This completes the proof.

\section{REFERENCES}

1. A. Friedman, Foundations of modern analysis, Holt, Rinehart and Winston, New York, 1970. MR 43 \#858.

2. S. Karlin and W. Studden, Tchebycheff systems: With applications in analysis and statistics, Pure and Appl. Math., Vol. 15, Interscience, New York, 1966. MR 34 \#4757.

3. R. Kelisky and T. J. Rivlin, Iterates of Bernstein polynomials, Pacific J. Math. 21 (1967), 511-520. MR 35 \#3328.

4. P. P. Korovkin, Linear operators and approximation theory, Fizmatgiz, Moscow, 1959; English transl., Russian Monographs and Texts on Advanced Math. and Phys., Vol. III, Gordon and Breach, New York; Hindustan, Delhi, 1960. MR 27 \#561.

5. G. G. Lorentz, Approximation of functions, Holt, Rinehart and Winston, New York, 1966. MR 35 \#4642.

6. D. Wulbert, Convergence of operators and Korovkin's theorem, J. Approximation Theory 1 (1968), 381-390. MR 38 \#3679.

Mathematical Sciences Department, IBM Watson Research Center, Yorktown HeIGHTS, New YORK 10598 